\title{
INVERSE SEMIGROUPS WITH ISOMORPHIC PARTIAL AUTOMORPHISM SEMIGROUPS
}

\author{
SIMON M. GOBERSTEIN
}

(Received 6 January 1988)

Communicated by P. G. Trotter

\begin{abstract}
It is shown that a so-called shortly connected combinatorial inverse semigroup is strongly latticedetermined "modulo semilattices". One of the consequences of this theorem is the known fact that a simple inverse semigroup with modular lattice of full inverse subsemigroups is strongly lattice-determined [7]. The partial automorphism semigroup of an inverse semigroup $S$ consists of all isomorphisms between inverse subsemigroups of $S$. It is proved that if $S$ is a shortly connected combinatorial inverse semigroup, $T$ an inverse semigroup and the partial automorphism semigroups of $S$ and $T$ are isomorphic, then either $S$ and $T$ are isomorphic or they are dually isomorphic chains (with respect to the natural partial order); moreover, any isomorphism between the partial automorphism semigroups of $S$ and $T$ is induced either by an isomorphism or, if $S$ and $T$ are dually isomorphic chains, by a dual isomorphism between $S$ and $T$. Counterexamples are constructed to demonstrate that the assumptions about $S$ being shortly connected and combinatorial are essential.
\end{abstract}

1980 Mathematics subject classification (Amer. Math. Soc.) (1985 Revision): 20 M 10, 20 M 18, $20 \mathrm{M} 20$.

\section{Introduction}

The purpose of this paper is to study to what extent an inverse semigroup $S$ is determined by its partial automorphism semigroup, which is defined as the inverse semigroup of all isomorphisms between inverse subsemigroups of $S$ (including the empty one). The partial automorphism semigroup can

(C) 1989 Australian Mathematical Society $0263-6115 / 89 \$ A 2.00+0.00$ 
be naturally defined for other mathematical structures as well. Several problems concerning partial automorphism semigroups of mathematical structures were posed by Preston in his well-known address [17]. Problem 4 of [17] deals specifically with the question of characterizing algebras by their partial automorphism semigroups. For several classes of groups and semigroups this problem has been considered in a number of publications (see [3] for a brief survey). However only a few results in that direction were obtained for the class of inverse semigroups other than groups. In [13] Libih proved that a monogenic inverse semigroup is determined up to isomorphism by its partial automorphism semigroup in the class of all inverse semigroups. In [14] several special types of commutative inverse semigroups determined by their partial automorphism semigroups were described.

It is known [1] that there exist nonisomorphic groups with isomorphic partial automorphism semigroups. Thus in the study of determinability of inverse semigroups by their partial automorphism semigroups, it is natural to concentrate on the class of combinatorial inverse semigroups (that is those which do not contain nontrivial subgroups). An isomorphism between partial automorphism semigroups of two inverse semigroups induces their lattice isomorphism (that is, an isomorphism between their lattices of inverse subsemigroups). Therefore in Section 1 we study lattice isomorphisms of combinatorial inverse semigroups. The main result here (Theorem 5) implies that a so-called shortly connected combinatorial inverse semigroup is strongly lattice-determined "modulo semilattices". One of the consequences of this theorem is the known fact that a simple inverse semigroup with modular lattice of full inverse subsemigroups is strongly lattice-determined [7]. In Section 2 we prove our principal result (Theorem 8 ) that if $S$ is a shortly connected combinatorial inverse semigroup, $T$ an inverse semigroup and the partial automorphism semigroups of $S$ and $T$ are isomorphic, then either $S \cong T$ or $S$ and $T$ are dually isomorphic chains (with respect to the natural partial order); moreover, any isomorphism between the partial automorphism semigroups of $S$ and $T$ is induced either by an isomorphism or, if $S$ and $T$ are dually isomorphic chains, by a dual isomorphism between $S$ and $T$. We also construct counterexamples to demonstrate that our assumptions about $S$ in Theorems 5 and 8 (that $S$ is shortly connected and combinatorial) are essential-if the first one is dropped or the second is weakened to the requirement that $S$ be fundamental, the theorems will no longer hold.

Preliminary versions of results from Section 2 were announced at the 1984 Marquette Conference on Semigroups and are described without proofs in [3]. 


\section{Lattice isomorphisms}

Let $S$ be an inverse semigroup. If $X$ is a subset of $S$, denote by $\langle X\rangle$ the inverse subsemigroup of $S$ generated by $X$. To indicate that $H$ is an inverse subsemigroup of $S$, we write $H \leq S$. It will be assumed that the empty set $\varnothing \leq S$. Then the set of all inverse subsemigroups of $S$, partially ordered by inclusion, is a lattice which will be denoted by $\operatorname{Sub}(S)$. Let $T$ be an inverse semigroup such that $\operatorname{Sub}(S) \cong \operatorname{Sub}(T)$. Then $S$ and $T$ are said to be lattice isomorphic and any isomorphism of $\operatorname{Sub}(S)$ onto $\operatorname{Sub}(T)$ is called a lattice isomorphism of $S$ onto $T$. If $\Phi$ is a lattice isomorphism of $S$ onto $T$, we will say that $\Phi$ is induced by a mapping $\phi: S \rightarrow T$ (or that $\phi$ induces $\Phi$ ) if $H \Phi=H \phi$ for any $H \leq S$. If $S$ is isomorphic to each inverse semigroup which is lattice isomorphic to $S$, then $S$ is called lattice-determined. We say that $S$ is strongly lattice-determined if each lattice isomorphism of $S$ onto an inverse semigroup $T$ is induced by an isomorphism of $S$ upon $T$. As usual, $E_{S}$ will denote the semilattice of idempotents of $S$ and $\leq$ the canonical partial order on $S$ (that is, $x \leq y$ if and only if $x=x x^{-1} y$ for $x, y \in S$ ). Recall that if $x$ and $y$ are elements of a certain poset, then $x \| y$ means that $x$ and $y$ are not comparable, and $x \sharp y$ denotes the negation of $x \| y$.

Result A (Jones [9, Lemma 1.1]). Let $S$ and $T$ be inverse semigroups and $\Phi$ a lattice isomorphism of $S$ onto $T$. Then there is a unique bijection $\phi_{E}$ of $E_{S}$ upon $E_{T}$ such that $\langle e\rangle \Phi=\left\langle e \phi_{E}\right\rangle$ for every $e \in E_{S}$, and the following conditions are satisfied for all $e, f \in E_{S}$ :

(i) $e \nVdash f$ if and only if $e \phi_{E} \nVdash f \phi_{E}$;

(ii) $e \| f$ implies $(e f) \phi_{E}=\left(e \phi_{E}\right)\left(f \phi_{E}\right)$.

It must be emphasized that $\phi_{E}$ is not, in general, an isomorphism of $E_{S}$ onto $E_{T}$ (see, for example, [9]).

If $X$ is a set, denote by $\Delta_{X}$ the equality (or "diagonal") relation on $X$. Recall [16] that a semigroup $S$ is said to be combinatorial if $\mathscr{C}=\Delta_{S}$. Clearly an inverse semigroup is combinatorial if and only if all its subgroups are trivial.

Result B (A corollary to [4, Lemma 2.1]). Let $S$ and $T$ be inverse semigroups and $\phi: S \rightarrow T$ a bijection such that $\left.\phi\right|_{E_{S}}$ is an isomorphism of $E_{S}$ onto $E_{T}$. If $S$ is combinatorial, then $\phi$ is an isomorphism of $S$ onto $T$ if and only if $\left(x^{-1}\right) \phi=(x \phi)^{-1}$ and $(e x) \phi=(e \phi)(x \phi)$ for any $x \in S$ and any $e \leq x x^{-1}$.

If an inverse semigroup $S$ is combinatorial and an inverse semigroup $T$ is lattice isomorphic to $S$, then $T$ is combinatorial as well [9, Corollary 1.3]. 
Through the rest of this section $S$ and $T$ are combinatorial inverse semigroups and $\Phi$ is a lattice isomorphism of $S$ onto $T$.

Result C (from Jones [12, Proposition 1.6 and Corollary 1.7]). For any $x \in S$, there exists a unique $y \in T$ such that $\langle x\rangle \Phi=\langle y\rangle,\left(x x^{-1}\right) \phi_{E}=y y^{-1}$ and $\left(x^{-1} x\right) \phi_{E}=y^{-1} y$. The mapping $\phi: x \mapsto y$ is a bijection of $S$ onto $T$ with the following properties:

(i) $\phi$ extends $\phi_{E}$, that is $\left.\phi\right|_{E_{S}}=\phi_{E}$;

(ii) $\phi$ and $\phi^{-1}$ preserve $\mathscr{L}$ - and $\mathscr{R}$-classes;

(iii) if $\theta$ is any homomorphism of $S$ onto $T$ which induces $\Phi$, then $\theta=\phi$.

We will say that $\phi$ is the $\Phi$-associated bijection of $S$ onto $T$. This bijection will be denoted by $\phi$ through the rest of this section.

It has long been known that any free group (in particular, the infinite cyclic group) is strongly lattice-determined [18]. More recently it was proved [9], [11], that any free inverse semigroup (in particular, the free monogenic one) and the bicyclic semigroup are also strongly lattice-determined. Furthermore, if in Result $\mathrm{A}, S$ is a monogenic inverse semigroup, then $T$ is also monogenic and $\phi_{E}$ is an isomorphism of $E_{S}$ onto $E_{T}[12$, Lemma 1.4 and Corollary 1.13]. Combining these results and [12, Lemma 3.6] with the well-known facts about the structure of monogenic inverse semigroups [16, Chapter IX], one can readily obtain the following theorem (thus even though no explicit proof of it, to the best of our knowledge, has been published yet, the result is undoubtedly true):

Result D (Ershova, see [20, Theorem I.39]). A monogenic inverse semigroup which contains no finite nontrivial subgroups is strongly latticedetermined.

REMARK 1. In what follows some of the basic results about the structure of monogenic inverse semigroups will be used without reference. The reader may consult [16, Chapter IX] for a detailed exposition.

As a consequence of Result $D$, we obtain

LEMMA 1. For any $a \in S,\left.\phi\right|_{\langle a\rangle}$ is an isomorphism of $\langle a\rangle$ onto $\langle a \phi\rangle$. In particular, $\left(a^{-1}\right) \phi=(a \phi)^{-1}$.

Proof. Let $a \in S, \Psi=\left.\Phi\right|_{\operatorname{Sub}(\langle a\rangle)}$ and $\psi=\left.\phi\right|_{\langle a\rangle}$. Then $\Psi$ is a lattice isomorphism and $\psi$ is the $\Psi$-associated bijection of $\langle a\rangle$ onto $\langle a \psi\rangle(=\langle a \phi\rangle)$. Since $\langle a\rangle$ is combinatorial, it is strongly lattice-determined, from Result D. Thus there exists an isomorphism of $\langle a\rangle$ onto $\langle a \psi\rangle$ which induces $\Psi$. By Result C(iii), this isomorphism must coincide with $\psi$. 
Let $U$ be an inverse semigroup, $a \in U$ and $e<a a^{-1}$. If there is no $f \in\langle a\rangle$ such that $e<f<a a^{-1}$, we say that $e$ is a-covered by $a a^{-1}$ and write $e \prec_{a} a a^{-1}$. The crucial result is the following.

LEMMA 2. If $\phi_{E}$ is an isomorphism of $E_{S}$ onto $E_{T}$, then for any $a \in S$ and $e \in E$ such that $e \prec_{a} a a^{-1},(e a) \phi=(e \phi)(a \phi)$.

Proof. Note that the properties (i) and (ii) of $\phi$ from Result $\mathrm{C}$ will be used below without comments. Let $a \in S$ and $e \in E_{S}$ be such that $e$ is $a$ covered by $a a^{-1}$. Suppose that $\phi_{E}$ is an isomorphism of $E_{S}$ onto $E_{T}$. Then, if $a \in E_{S}$, we have $(e a) \phi=(e \phi)(a \phi)$. Thus from now on we will assume that $a \notin E_{S}$ (since $S$ is combinatorial, this means that $a a^{-1} \neq a^{-1} a$ ).

Since $e \mathscr{R} e a$, we have $e \phi \mathscr{R}(e a) \phi$. Moreover,

$$
(e \phi)(a \phi)[(e \phi)(a \phi)]^{-1}=(e \phi)(a \phi)(a \phi)^{-1}=(e \phi) \cdot\left(a a^{-1}\right) \phi=\left(e a a^{-1}\right) \phi=e \phi \text {, }
$$

so that $e \phi \mathscr{R}(e \phi)(a \phi)$. It follows that $(e \phi)(a \phi)=s \phi$ for some $s \in R_{e}$. Since $\langle e, a\rangle \Phi=\langle e \phi\rangle \vee\langle a \phi\rangle=\langle e \phi, a \phi\rangle$ and $(e \phi)(a \phi) \in\langle e \phi, a \phi\rangle$, we have $s \in\langle e, a\rangle$. Suppose that $s=x u y$ where $x, y, u \in\langle e, a\rangle$ and $u=u^{2}$. Then $s=\left(x u x^{-1}\right)(x y)$. Repeating this as many times as necessary, we can write $s$ in the form $s=g t$ where $g=g^{2} \in\langle e, a\rangle, t \in\langle a\rangle$ and no subword of $t$ (considered as a word in $a$ and $a^{-1}$ ) is an idempotent (cf. [10, Lemma 2.1]). Thus either $t=a^{n}$ or $t=a^{-n}$ for some integer $n \geq 1$. Moreover, e⿻⿱R乛s $=e s=e g t$. It follows that $R_{e}=R_{e g t} \leq R_{e g} \leq R_{e}$, whence $e g=e$ and $s=(e g) t=e t$.

CASE I. $a a^{-1} \nless a^{-1} a$.

(1) $s=e a^{-n}$ for $n \geq 1$.

We have

$$
e \mathscr{R} e a^{-n} \mathscr{R} e a^{-1} \mathscr{R}\left(e a^{-1}\right)\left(e a^{-1}\right)^{-1}=e a^{-1} a .
$$

Thus $e=e a^{-1} a \leq a^{-1} a$. Since $e<a a^{-1}$, it follows that $e \leq\left(a^{-1} a\right)\left(a a^{-1}\right)<$ $a a^{-1}$. However $e$ is $a$-covered by $a a^{-1}$. Hence $e=\left(a^{-1} a\right)\left(a a^{-1}\right) \in\langle a\rangle$ and, by Lemma $1,(e a) \phi=(e \phi)(a \phi)$.

(2) $s=e a^{n}$ for $n \geq 1$.

If $n=1$, then $(e \phi)(a \phi)=s \phi=(e a) \phi$. Now suppose that $n \geq 2$. Then $(e \phi)(a \phi)=\left(e a^{n}\right) \phi$ and we have

$$
\left(a^{-n} e a^{n}\right) \phi=\left[\left(e a^{n}\right)^{-1}\left(e a^{n}\right)\right] \phi=\left[\left(e a^{n}\right) \phi\right]^{-1} \cdot\left(e a^{n}\right) \phi=(a \phi)^{-1}(e \phi)(a \phi) .
$$

Since $n \geq 2, a^{-n} e a^{n} \leq a^{-n} a^{n} \leq a^{-2} a^{2}$. Thus

$$
(a \phi)^{-1}(e \phi)(a \phi)=\left(a^{-n} e a^{n}\right) \phi \leq\left(a^{-2} a^{2}\right) \phi,
$$

whence

$$
\begin{aligned}
e \phi & =(a \phi)(a \phi)^{-1}(e \phi)(a \phi)(a \phi)^{-1} \leq(a \phi)(a \phi)^{-2}(a \phi)^{2}(a \phi)^{-1} \\
& =\left[\left(a a^{-1}\right)\left(a^{-1} a\right)\right] \phi .
\end{aligned}
$$


Therefore, $e \leq\left(a a^{-1}\right)\left(a^{-1} a\right)<a a^{-1}$. Since $e \prec_{a} a a^{-1}$, it follows that $e=\left(a a^{-1}\right)\left(a^{-1} a\right) \in\langle a\rangle$ and again, by Lemma $1,(e a) \phi=(e \phi)(a \phi)$.

CASE II. $a a^{-1}<a^{-1} a$.

Let $b=a^{-1}$ and $f=a^{-1} e a$. Then $f \prec_{b} b b^{-1}$ and by case $\mathrm{I},(f b) \phi=$ $(f \phi)(b \phi)$. It follows that

$$
\begin{aligned}
(e a) \phi & =\left(b^{-1} f b \cdot b^{-1}\right) \phi=\left(b^{-1} f\right) \phi=[(f b) \phi]^{-1}=[(f \phi)(b \phi)]^{-1} \\
& =(b \phi)^{-1}(f \phi)(b \phi) \cdot(b \phi)^{-1}=[(f \phi)(b \phi)]^{-1}(f \phi)(b \phi) \cdot(b \phi)^{-1} \\
& =[(f b) \phi]^{-1} \cdot(f b) \phi \cdot\left(b^{-1} \phi\right)=\left(b^{-1} f b\right) \phi \cdot\left(b^{-1} \phi\right)=(e \phi)(a \phi) .
\end{aligned}
$$

This completes the proof.

Now we will describe a class of inverse semigroups which will play an important role in this paper. Let $U$ be an inverse semigroup. Take $a \in U$ and $e<a a^{-1}$. Suppose that for some $n \geq 1$, there exist $e_{0}, e_{1}, \ldots, e_{n} \in E_{U}$ such that $e=e_{0}<e_{1}<\cdots<e_{n}=a a^{-1}$ and for any $i=1, \ldots, n, e_{i-1}$ is $a_{i^{-}}$ covered by $e_{i}$ where $a_{i}=e_{i} a$ (and hence $\left.a_{i} a_{i}^{-1}=e_{i}\right)$. Then $\left(e_{0}, e_{1}, \ldots, e_{n}\right)$ will be called a short bypass from $e$ to $a a^{-1}$. If for any $a \in U$ and any $e<a a^{-1}$, there exists a short bypass from $e$ to $a a^{-1}, U$ is said to be shortly connected. It is obvious that any semilattice and any finite inverse semigroup are shortly connected. In fact, semilattices and finite inverse semigroups are contained in a large subclass of shortly connected inverse semigroups described below.

Let $a \in U$ and $e<a a^{-1}$. A short bypass $\left(e, f_{1}, \ldots, f_{k}\right)$ from $e$ to $f_{k}=a a^{-1}$ is called a short link between $e$ and $a a^{-1}$ if for any $i=1, \ldots, k-1$ (when $k>1), f_{i} \in\langle a\rangle, f_{i}$ is covered by $f_{i+1}$ in $(\langle a\rangle, \leq)$ and there is no $g \in E_{\langle a\rangle}$ such that $e<g<f_{1}$. If for all $a \in U$ and $e<a a^{-1}$, there is a short link between $e$ and $a a^{-1}$, then $U$ is called shortly linked. It is immediate from the definition that a shortly linked inverse semigroup is shortly connected.

The following facts about the semilattice of idempotents of a monogenic inverse semigroup are explicitly presented in [2] (they can also be recovered from [16]). Let $U$ be an inverse semigroup and $a \in U$. If $\langle a\rangle$ is a free monogenic inverse semigroup, then $\left(E_{\langle a\rangle}, \leq\right)$ is isomorphic to the direct product $C_{\omega} \times C_{\omega}$ from which the largest element is deleted (here $C_{\omega}$ denotes an $\omega$ chain [16], that is, the set of nonnegative integers ordered dually to their natural order). Hence in this case $\left(E_{\langle a\rangle}, \leq\right)$ has the following diagram

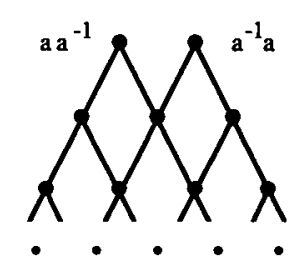

Figure 1 
If $\langle a\rangle$ is not free, then the diagram of $\left(E_{\langle a\rangle}, \leq\right)$ is one of the following

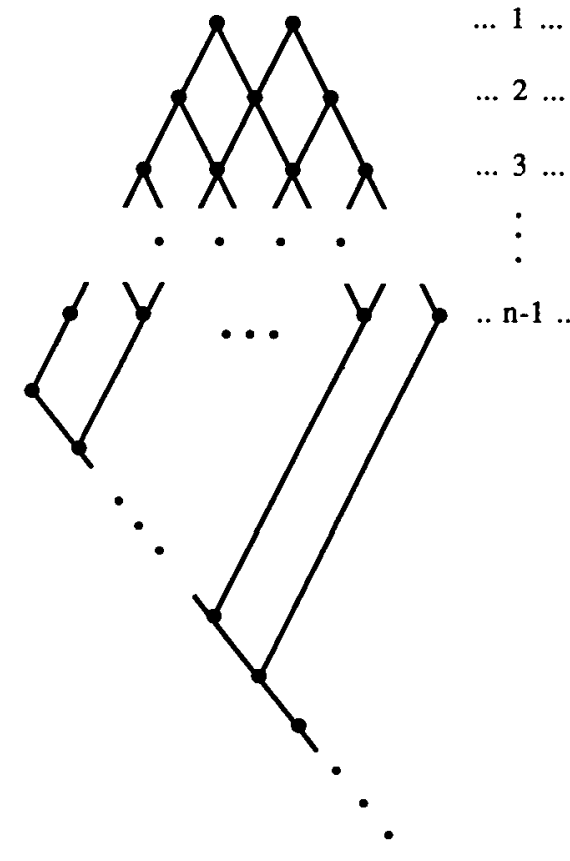

(a)
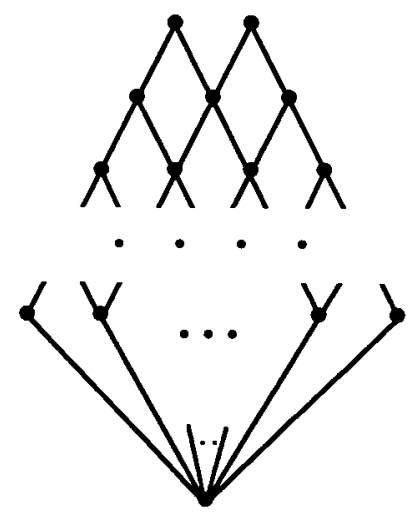

(b)

FiguRE 2

The diagram 2(a) corresponds to the case when $\langle a\rangle$ has a kernel which is a bicyclic semigroup, and the diagram 2 (b) to the case where the kernel of $\langle a\rangle$ is a (cyclic) group. The numbers $1, \ldots, n-1$ indicate the depth (or the weight [16]) of idempotents of $\langle a\rangle$ which do not belong to the kernel. Note that $n \geq 1$; if $n=1$, then $\langle a\rangle$ coincides with the kernel.

Using this information about the structure of $\left(E_{\langle a\rangle}, \leq\right)$ we can establish the following proposition which, in particular, gives a convenient description of shortly linked inverse semigroups. For $a \in U$ and $e<a a^{-1}$, set $F_{e, a}=$ $\left\{f \in\langle a\rangle: e<f \leq a a^{-1}\right\}$.

Proposition 3. Let $U$ be an inverse semigroup, $a \in U$ and $e<a a^{-1}$. Then the following conditions are equivalent:

(i) there is a short bypass $\left(e, e_{1}, \ldots, e_{l}\right)$ from e to $a a^{-1}=e_{l}$ such that $e_{i} \in\langle a\rangle$ for any $i=1, \ldots, l$;

(ii) there is a short link between e and $a a^{-1}$;

(iii) if $e \notin\langle a\rangle$, then $F_{e, a}$ has a least element;

(iv) $F_{e, a}$ is finite. 
Thus $U$ is shortly linked if and only if for any $a \in U$ and $e<a a^{-1}, F_{e, a}$ is finite.

Proof. (i) $\Rightarrow$ (ii). Let $\left(e, e_{1}, \ldots, e_{l}\right)$ be a short bypass from $e$ to $a a^{-1}=e_{l}$ such that $e_{i} \in\langle a\rangle$ for any $i=1, \ldots, l$. Suppose that this bypass is not a short link between $e$ and $a a^{-1}$. If $l>1$ and for some $1 \leq i \leq l-1, e_{i}$ is not covered in $(\langle a\rangle, \leq)$ by $e_{i+1}$, since the segment $\left[e_{i}, e_{i+1}\right]$ of $(\langle a\rangle, \leq)$ is finite, we can find $g_{1}, \ldots, g_{k} \in\left[e_{i}, e_{i+1}\right]$ such that $e_{i} \prec g_{1} \prec \cdots \prec g_{k} \prec e_{i+1}$ (here and later in the proof $\prec$ denotes the covering relation in $(\langle a\rangle, \leq))$. Similarly "filling up" other "gaps" in the chain $e_{1}<\cdots<e_{l}$ (if they exist), we will get a short bypass $\left(e, f_{1}, \ldots, f_{k}\right)$ from $e$ to $a a^{-1}=f_{k}$ in which for every $i=1, \ldots, k-1$ (if $k>1$ ), $f_{i} \in\langle a\rangle$ and $f_{i} \prec f_{i+1}$.

Set $a_{1}=f_{1} a$ and recall that $e$ is $a_{1}$-covered by $a_{1} a_{1}^{-1}=f_{1}$. Let $\left(e, f_{1}\right]_{a}=$ $\left\{h \in E_{\langle a\rangle}: e<h \leq f_{1}\right\}$. Suppose that $\left(e, f_{1}\right]_{a}$ is infinite. Then the diagram of $E_{\langle a\rangle}$ is given either by Figure 1 or by Figure 2(a), and there exists an infinite family $\left\{h_{n} \in\left(e, f_{1}\right]_{a}: n \geq 0\right\}$ such that $f_{1}=h_{0} \succ h_{1} \succ h_{2} \succ \cdots \succ h_{n} \succ \cdots$. In the first case, $E_{\langle a\rangle}$ contains a sublattice

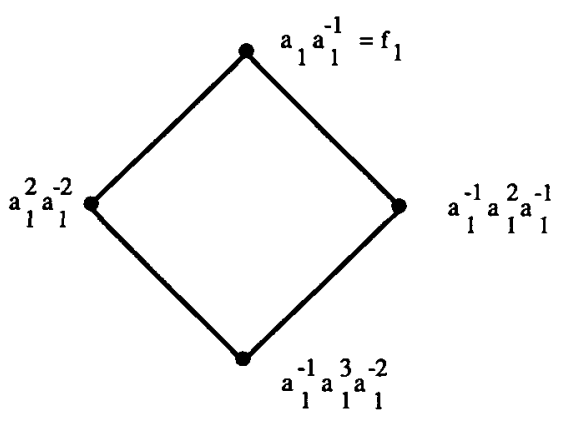

and for some $n \geq 1$, either $a_{1}^{2} a_{1}^{-2} \geq h_{n}>e$ or $a_{1}^{-1} a_{1}^{2} a_{1}^{-1} \geq h_{n}>e$; a contradiction. In the second case, take any $g \in E_{\left\langle a_{1}\right\rangle}$ such that $g<f_{1}$ and $g$ belongs to the kernel of $\langle a\rangle$. Then for some $n \geq 1, g \geq h_{n}>e$; again a contradiction. Thus $\left(e, f_{1}\right]_{a}$ is finite and we can find $g_{1}^{\prime}, \ldots, g_{m}^{\prime} \in\left(e, f_{1}\right]_{a}$ such that $e<g_{1}^{\prime} \prec \cdots \prec g_{m}^{\prime}=f_{1}$ and $g_{1}^{\prime}$ is a minimal element in $\left(e, f_{1}\right]_{a}$. Therefore $\left(e, g_{1}^{\prime}, \ldots, g_{m}^{\prime}=f_{1}, \ldots, f_{k}\right)$ is a short link between $e$ and $a a^{-1}=f_{k}$.

(ii) $\Rightarrow$ (iii). Suppose that $e \notin\langle a\rangle$ and let $\left(e, f_{1}, \ldots, f_{m}\right)$ be a short link between $e$ and $a a^{-1}=f_{m}$. Take any $g \in F_{e, a}$. If $f_{1} \$ g$, then $f_{1}>f_{1} g>e$, a contradiction. Thus $f_{1} \leq g$, that is $f_{1}$ is the least element in $F_{e, a}$.

(iii) $\Rightarrow$ (iv). Since $F_{e, a}$ consists of all elements of $E a a^{-1}$ which are greater than or equal to some element of $E_{\langle a\rangle}, F_{e, a}$ is finite.

(iv) $\Rightarrow$ (i). Suppose that $F_{e, a}$ is finite. Let $e_{1}$ be a minimal element in $F_{e, a}$ and let $a_{1}=e_{1} a$. Then $e$ is $a_{1}$-covered by $e_{1}$. Furthermore, there exist 
$e_{2}, \ldots, e_{l} \in\langle a\rangle$ (if $e_{1} \neq a a^{-1}$ ) such that $e_{1} \prec e_{2} \prec \ldots \prec e_{l}=a a^{-1}$. Then $\left(e, e_{1}, \ldots, e_{l}\right)$ is the desired short bypass from $e$ to $a a^{-1}$.

Let $U$ be an inverse semigroup. If for every $e \in E_{U}$ and $a \in U$ such that $a a^{-1}>a^{-1} a, a^{-n} a^{n}<e$ for some $n \geq 1$, then $E_{U}$ is called Archimedean in $U$ (see, for example, [10] or [11]). An immediate consequence of Proposition 3 is the following

COROLlary 4. Let $U$ be an inverse semigroup. If for every $e \in E_{U}$ and every element $a \in U$ of infinite order, there exists $g \in E_{\langle a\rangle}$ such that $g<e$, then $U$ is shortly linked. In particular, $U$ is shortly linked if each nonidempotent of $U$ is either of finite order, or generates a bicyclic subsemigroup of $U$ and $E_{U}$ is Archimedean in $U$.

The following theorem is the main result of this section. To make its formulation complete, we repeat the assumptions made earlier about $S, T$ and $\Phi$.

TheOREM 5. Let $S$ be a combinatorial inverse semigroup, $T$ an inverse semigroup and $\Phi$ a lattice isomorphism of $S$ onto $T$. Let $\phi$ be the $\Phi$-associated bijection of $S$ upon $T$ and $\phi_{E}=\left.\phi\right|_{E_{S}}$. Suppose that $S$ is shortly connected and $\phi_{E}$ is an isomorphism of $E_{S}$ onto $E_{T}$. Then $\phi$ is the unique isomorphism of $S$ onto $T$ which induces $\Phi$.

Proof. Take any $a \in S$ and $e \in E_{S}$ such that $e \leq a a^{-1}$. If $e=a a^{-1}$, then $(e a) \phi=a \phi=(a \phi)(a \phi)^{-1}(a \phi)=\left(a a^{-1}\right) \phi \cdot(a \phi)=(e \phi)(a \phi)$ by Result $\mathrm{C}$ (alternatively we could refer to Lemma 1). Now suppose that $e<a a^{-1}$. Since $S$ is shortly connected, there exists a short bypass $\left(e_{0}, e_{1}, \ldots, e_{n}\right)$ from $e$ to $a a^{-1}$. Recall that this means that $e=e_{0}<e_{1}<\cdots<e_{n}=a a^{-1}$ and for any $i=1, \ldots, n, e_{i-1}$ is $a_{i}$-covered by $a_{i} a_{i}^{-1}$ where $a_{i}=e_{i} a$. Note that for $i=1, \ldots, n-1, a_{i}=e_{i} a_{i+1}$. Using Lemma 2 , we get

$$
\begin{aligned}
(e a) \varphi & =\left(e_{0} a_{1}\right) \phi=\left(e_{0} \varphi\right)\left(a_{1} \varphi\right)=\left(e_{0} \varphi\right)\left(e_{1} a_{2}\right) \varphi=\cdots \\
& =\left(e_{0} \varphi\right) \cdot\left(e_{n-1} a_{n}\right) \phi=\left(e_{0} \varphi\right)\left(e_{n-1} \phi\right)\left(a_{n} \varphi\right)=(e \varphi)(a \varphi) .
\end{aligned}
$$

(Of course, we could have used a simple induction argument here.) By Lemma $1,\left(a^{-1}\right) \phi=(a \phi)^{-1}$. Therefore, according to Result $\mathbf{B}, \phi$ is an isomorphism of $S$ onto $T$. The fact that $\phi$ induces $\Phi$ is obvious. By Result C(iii) $\phi$ is the only isomorphism of $S$ upon $T$ inducing $\Phi$.

Theorem 5 states that any shortly connected combinatorial inverse semigroup is strongly lattice-determined "modulo semilattices". In the next section it will be shown that there exist nonisomorphic combinatorial, but not shortly connected, inverse semigroups $S$ and $T$ which are lattice isomorphic 
and such that $E_{S} \cong E_{T}$. Thus the condition that $S$ is shortly connected cannot be dropped from the formulation of Theorem 5 .

Recall [6], [16] that an inverse semigroup $U$ is said to be fundamental if $\Delta_{U}$ is the only congruence on $U$ contained in $\mathscr{H}$. It will be also shown in the next section that the assumption in Theorem 5 that $S$ is combinatorial cannot be replaced by a less restrictive assumption that $S$ is fundamental (even when $S$ is finite) without invalidating the conclusion of the theorem.

REMARK 2. It ought to be mentioned that certain fundamental inverse semigroups are lattice-determined even if they are not shortly connected. As an illustration consider the following. Recall that if $E$ is a semilattice, then the Munn semigroup $T_{E}$ is an inverse semigroup (under composition) consisting of all isomorphisms between principal ideals of $E$. The semilattice of idempotents of $T_{E}$ is isomorphic to $E$ and an inverse semigroup $U$ with $E_{U} \cong E$ is fundamental if and only if $U$ is isomorphic to a full (i.e., containing all the idempotents) inverse subsemigroup of $T_{E}$ (see [6, Section V.4]). According to Proposition 2.2 of [12], if $E$ is a semilattice such that $T_{E}$ is simple and each principal ideal of $E$ has a finite group of automorphisms (the latter condition is, of course, equivalent to saying that each $\mathscr{H}$-class of $T_{E}$ is finite), then $T_{E}$ is lattice-determined. With only a slight change in the proof of that proposition we can establish the following more general result:

Let $E$ be any semilattice such that the group of automorphisms of each principal ideal of $E$ is finite. Let $U$ be an inverse semigroup with $E_{U} \cong E$. If $\operatorname{Sub}(U) \cong \operatorname{Sub}\left(T_{E}\right)$, then $U \cong T_{E}$.

An inverse semigroup $U$ is called modular [distributive] if the lattice of all full inverse subsemigroups of $U$ is modular [distributive]. The structure of simple distributive and of simple modular inverse semigroups was described in [10] and in [8] respectively. In [11] Jones proved that a simple distributive inverse semigroup, which is not a group, is strongly lattice-determined. A recent paper by Johnston extended this theorem to simple modular inverse semigroups [7]. We conclude this section by showing that the latter (and thus the former) result is a consequence of Theorem 5 . We will need the following

Result E (from Johnston and Jones [8, Propositions 2.2, 2.5 and 4.3(iii)]). Let $S$ be a simple modular inverse semigroup which is not a group. Then

(i) $S$ is combinatorial;

(ii) a nonidempotent of $S$ is either of finite order or generates a bicyclic subsemigroup of $S$;

(iii) $E_{S}$ is Archimedean in $S$.

Now we can easily deduce the following corollary. 
COROLlary 6 [7, TheOREM 15]. Let $S$ be a simple modular inverse semigroup, which is not a group, and $T$ an inverse semigroup. Then each isomorphism of $\operatorname{Sub}(S)$ onto $\operatorname{Sub}(T)$ is induced by a unique isomorphism of $S$ upon $T$.

Proof. By Result E(i) $S$ is combinatorial, and by Corollary 4 and Result $\mathrm{E}$, (ii) and (iii), $S$ is shortly linked. Let $\Phi$ be a lattice isomorphism of $S$ onto $T, \phi$ the $\Phi$-associated bijection of $S$ upon $T$ and $\phi_{E}=\left.\phi\right|_{E_{S}}$. Since $S$ is simple, it follows from [12, Lemma 1.10] that $\phi_{E}$ is an isomorphism of $E_{S}$ onto $E_{T}$. Thus, by Theorem $5, \phi$ is a unique isomorphism of $S$ upon $T$ which induces $\Phi$.

REMARK 3. The author initially introduced the class of shortly connected inverse semigroups and formulated and proved Lemma 2 and Theorem 5 , the central results of this section, using the following definition of $a$-covering: for an inverse semigroup $U$ and $e, a \in U$ with $e<a a^{-1}, e$ is said to be $a$-covered by $a a^{-1}$ if there is no $f \in\langle e, a\rangle$ satisfying $e<f<a a^{-1}$. P. R. Jones noticed that the author would have to change absolutely nothing in the formulations and proofs of Lemma 2 and Theorem 5 if in the definition of $a$-covering "no $f \in\langle e, a\rangle$ " is replaced by "no $f \in\langle a\rangle$ " (so that Theorem 5 would apply to a larger than the original class of shortly connected inverse semigroups), and observed that [7, Theorem 15] can be easily deduced from that more general statement of Theorem 5. The author is grateful to Dr. Jones for that important comment and for his kind suggestion to include Corollary 6 in this paper.

\section{2. $\mathscr{P} \mathscr{A}$-isomorphisms}

Let $S$ be an inverse semigroup. A partial automorphism of $S$ is defined here as an isomorphism between any two (isomorphic) inverse subsemigroups of $S$ (in particular, the empty set $\varnothing$ is a partial automorphism of $S$ ). The set of all partial automorphisms of $S$ with respect to composition is itself an inverse semigroup which will be denoted by $\mathscr{P} \mathscr{A}(S)$ and called the partial automorphism semigroup of $S$. It is clear that $\mathscr{P} \mathscr{A}(S) \leq \mathscr{I}(S)$ where $\mathscr{I}(S)$ is the symmetric inverse semigroup on the set $S$. Thus the idempotents of $\mathscr{P} \mathscr{A}(S)$ are precisely the partially identical mappings $\Delta_{H}$ of $S$ for every $H \leq S$ (note that $\Delta_{\varnothing}=\varnothing$ ). Since $\Delta_{H} \circ \Delta_{K}=\Delta_{H \cap K}$ for any $H, K \leq S$, the semilattice of idempotents of $\mathscr{P} \mathscr{A}(S)$ is isomorphic to $\operatorname{Sub}(S)$. Furthermore, the group of units of $\mathscr{P A}(S)$ is $\operatorname{Aut}(S)$, the automorphism group of $S$.

Let $S$ and $T$ be inverse semigroups. If $\mathscr{P} \mathscr{A}(S) \cong \mathscr{P} \mathscr{A}(T)$, then $S$ and $T$ are said to be $\mathscr{P} \mathscr{A}$-isomorphic and any isomorphism of $\mathscr{P} \mathscr{A}(S)$ upon $\mathscr{P} \mathscr{A}(T)$ 
is called a $\mathscr{P} \mathscr{A}$-isomorphism of $S$ onto $T$. Let $\Phi$ be a $\mathscr{P} \mathscr{A}$-isomorphism of $S$ onto $T$. For any $H \leq S$, define $H \Phi^{*}$ by the formula $\Delta_{H} \Phi=\Delta_{H \Phi^{*}}$. Then $\Phi^{*}$ is a lattice isomorphism of $S$ onto $T$ (defined by $\Phi$ ). It is also clear that the restriction of $\Phi$ to $\operatorname{Aut}(S)$ is an isomorphism of $\operatorname{Aut}(S)$ onto $\operatorname{Aut}(T)$. On the other hand it is not hard to find examples of inverse semigroups with isomorphic groups of automorphisms and lattices of inverse subsemigroups which nevertheless are not $\mathscr{P} \mathscr{A}$-isomorphic $\left(\mathbf{Z}_{35}\right.$ and $\mathbf{Z}_{39}$ represent perhaps the simplest such example [15]).

LEMMA 7. Let $S$ and $T$ be inverse semigroups and $\Phi$ a $\mathscr{P} \mathscr{A}$-isomorphism of $S$ onto $T$. Then for any $\alpha \in \mathscr{P} \mathscr{A}(S), \operatorname{dom}(\alpha \Phi)=[\operatorname{dom}(\alpha)] \Phi^{*}$ and $\operatorname{ran}(\alpha \Phi)=$ $[\operatorname{ran}(\alpha)] \Phi^{*}$.

Proof. Let $\alpha \in \mathscr{P} \mathscr{A}(S)$ and $H=\operatorname{dom}(\alpha), K=\operatorname{ran}(\alpha)$. Then $\alpha \circ \alpha^{-1}=\Delta_{H}$ and $\alpha^{-1} \circ \alpha=\Delta_{K}$. It follows that $(\alpha \Phi) \circ(\alpha \Phi)^{-1}=\Delta_{H \Phi^{*}}$ and $(\alpha \Phi)^{-1} \circ(\alpha \Phi)=$ $\Delta_{K \Phi^{*}}$. Hence $\operatorname{dom}(\alpha \Phi)=H \Phi^{*}$ and $\operatorname{ran}(\alpha \Phi)=K \Phi^{*}$.

We say that a $\mathscr{P} \mathscr{A}$-isomorphism $\Phi$ of $S$ onto $T$ is induced by a bijection $\phi: S \rightarrow T$ (or that $\phi$ induces $\Phi$ ) if for every $\alpha \in \mathscr{P} \mathscr{A}(S), \alpha \Phi=\phi^{-1}$ 。 $\alpha \circ \phi$, that is, for any $x, y \in S, x \alpha=y$ if and only if $(x \phi)(\alpha \Phi)=y \phi$. If inverse semigroups are isomorphic, then any isomorphism between them obviously induces a $\mathscr{P} \mathscr{A}$ isomorphism. An inverse semigroup $S$ is called $\mathscr{P} \mathscr{A}$-determined if for any inverse semigroup $T, \mathscr{P} \mathscr{A}(S) \cong \mathscr{P} \mathscr{A}(T)$ if and only if $S \cong T$. If moreover any $\mathscr{P} \mathscr{A}$-isomorphism of $S$ onto $T$ is induced by an isomorphism of $S$ upon $T$, then $S$ is said to be strongly $\mathscr{P} \mathscr{A}$-determined.

Let $E$ be a semilattice and $\leq$ the natural partial order on $E$ (that is, for any $e, f \in E, e \leq f$ if and only if $e=e f$ ). Then by $E^{d}$ we denote the semilattice dual to $E$, that is, the underlying set of $E^{d}$ is $E$ and its natural partial order $\leq^{d}$ is dual to $\leq$.

RESULT F (a corollary to Schein [19]). Let $E$ be a semilattice and $F$ an inverse semigroup. Then $\mathscr{P} \mathscr{A}(E) \cong \mathscr{P} \mathscr{A}(F)$ if an only if $E \cong F$ or $(E, \leq)$

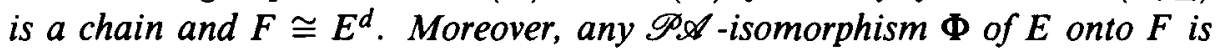
induced by a bijection $\phi_{E}$ defined by $\langle e\rangle \Phi^{*}=\left\langle e \phi_{E}\right\rangle$ for every $e \in E$, and $\phi_{E}$ is either an isomorphism of $E$ onto $F$ or, if $E$ is a chain and $F \cong E^{d}$, a dual isomorphism of $E$ upon $F$.

In addition, it is easy to see that if a bijection $\gamma: E \rightarrow F$ induces a $\mathscr{P} \mathscr{A}$ isomorphism $\Phi$ of $E$ onto $F$, then $\gamma=\phi_{E}$, that is, $\phi_{E}$ in Result $F$ is uniquely defined by $\Phi$.

Using Result $F$ and Theorem 5 , we will now show that any shortly connected combinatorial inverse semigroup $S$ is strongly $\mathscr{P} \mathscr{A}$-determined (unless $(S, \leq)$ is a chain which is not isomorphic to its dual). More precisely, we have 
THEOREM 8. Let $S$ be a shortly connected combinatorial inverse semigroup and $T$ an inverse semigroup. Then $\mathscr{P} \mathscr{A}(S) \cong \mathscr{P} \mathscr{A}(T)$ if and only if either $S \cong T$ or $(S, \leq)$ and $(T, \leq)$ are dually isomorphic chains. Moreover, any $\mathscr{P} \mathscr{A}$ isomorphism of $S$ onto $T$ is induced by a unique isomorphism of $S$ upon $T$ or, if $(S, \leq)$ is a chain and $T \cong S^{d}$, by a unique dual isomorphism of $S$ upon $T$.

Proof. Let $\Phi$ be a $\mathscr{P} \mathscr{A}$-isomorphism of $S$ onto $T$. The restriction of $\Phi$ to $\mathscr{P} \mathscr{A}\left(E_{S}\right)$ is a $\mathscr{P} \mathscr{A}$-isomorphism of $E_{S}$ upon $E_{T}$. By Result F, $\left.\Phi\right|_{\mathscr{P} \mathscr{A}\left(E_{S}\right)}$ is induced by the bijection $\phi_{E}: E_{S} \rightarrow E_{T}$ defined by $\langle e\rangle \Phi^{*}=\left\langle e \phi_{E}\right\rangle$ for every $e \in E_{S}$. From Results $\mathrm{A}$ and $\mathrm{C}$, it follows that this bijection $\phi_{E}$ is the restriction to $E_{S}$ of the $\Phi^{*}$-associated bijection of $S$ onto $T$. Moreover, according to Result $\mathrm{F}$, either $\phi_{E}$ is an isomorphism of $E_{S}$ onto $E_{T}$, or $\left(E_{S}, \leq\right)$ and $\left(E_{T}, \leq\right)$ are chains and $\phi_{E}$ is a dual isomorphism of $E_{S}$ onto $E_{T}$.

Suppose first that $\phi_{E}$ maps $E_{S}$ isomorphically onto $E_{T}$. Let $\phi$ denote the $\Phi^{*}$-associated bijection of $S$ onto $T$. Then by Theorem $5, \phi$ is the unique isomorphism of $S$ upon $T$ which induces $\Phi^{*}$. To show that $\phi$ induces $\Phi$, take an arbitrary $\alpha \in \mathscr{P} \mathscr{A}(S)$ and any $x, y \in S$ such that $x \alpha=y$. Set $\bar{\alpha}=\left.\alpha\right|_{\langle x\rangle}$. Then $\bar{\alpha} \in \mathscr{P} \mathscr{A}(S), \operatorname{dom}(\bar{\alpha})=\langle x\rangle$ and $\operatorname{ran}(\bar{\alpha})=\langle y\rangle$. By Lemma 7 and Result $\mathrm{C}, \operatorname{dom}(\bar{\alpha} \Phi)=\langle x\rangle \Phi^{*}=\langle x \phi\rangle$ and $\operatorname{ran}(\bar{\alpha} \Phi)=\langle y\rangle \Phi^{*}=\langle y \phi\rangle$. Hence $(x \phi)(\bar{\alpha} \Phi) \in\left\{y \phi,(y \phi)^{-1}\right\}$. Using Results $\mathrm{C}$ and $\mathrm{F}$, we get $\left[(x \phi)(x \phi)^{-1}\right](\bar{\alpha} \Phi)=$ $(y \phi)(y \phi)^{-1}$. Since $\bar{\alpha} \Phi$ is an isomorphism of $\langle x \phi\rangle$ onto $\langle y \phi\rangle$, it follows that $(x \phi)(\bar{\alpha} \Phi)=y \phi$ and hence $(x \phi)(\alpha \Phi)=y \phi$. Thus $\phi$ induces $\Phi$. If $\gamma$ is any isomorphism of $S$ onto $T$ which induces $\Phi$, then it is easy to see that $\gamma$ induces $\Phi^{*}$ and, by Result C(iii), $\gamma=\phi$, that is, $\phi$ is the only isomorphism of $S$ upon $T$ inducing $\Phi$.

Now assume that $\left(E_{S}, \leq\right)$ and $\left(E_{T}, \leq\right)$ are chains and $\phi_{E}$ is a dual isomorphism of $E_{S}$ onto $E_{T}$. We will show that in this case $S=E_{S}$ and $T=E_{T}$. Suppose that $S$ contains a bicyclic subsemigroup $\langle x\rangle$. Then the idempotents of $\langle x\rangle \Phi^{*}$ are well-ordered, so that $\langle x\rangle \Phi^{*}$ is a Clifford semigroup [6, Section V.5] which must be a chain since $T$ is combinatorial. Hence, by Result $\mathrm{A},\langle x\rangle$ is a chain; a contradiction. Thus $S$ is completely semisimple, that is idempotents in each $\mathscr{D}$-class of $S$ are incomparable. Since $S$ is combinatorial and $E_{S}$ is a chain, it follows that each $\mathscr{D}$-class of $S$ consists of just one idempotent, so that $S=E_{S}$. By symmetry (or by Result A) we also have $T=E_{T}$. To complete the proof, it remains to apply Result $F$ and the remark that follows it.

We are going to show that Theorem 8 need not hold for a combinatorial inverse semigroup $S$ which is not shortly connected, even if we assume in addition that $S$ is completely semisimple.

EXAMPLE 1. Let $A=\langle a\rangle$ be the free monogenic inverse semigroup generated by $a$, and let $E_{1}=E_{A}$ (the diagram of $E_{1}$ is given by Figure 1). For 
any $e \leq a a^{-1}$, set $e \theta_{a}=a^{-1} e a$. Then [6, Section V.4] $\theta_{a}$ is an isomorphism of $E_{1} a a^{-1}$ onto $E_{1} a^{-1} a$, so that $\theta_{a} \in T_{E_{1}}$. Let $E_{0}=\left\{0, e_{0}, e_{1}, e_{2}\right\}$ be a fourelement primitive semilattice (that is, 0 is a zero of $E_{0}$ and $e_{i} e_{j}=0$ if $i \neq j$ ) disjoint from $E_{1}$. Let $\bar{E}$ be the ordinal sum of $E_{0}$ and $E_{1}$, that is, $\bar{E}=E_{0} \cup E_{1}$ and the natural partial order $\leq$ on $\bar{E}$ is such that $e<f$ for all $e \in E_{0}$ and $f \in E_{1}$, and its restriction to $E_{i}$ coincides with the natural partial order on $E_{i}(i=0,1)$. A diagram of $\bar{E}$ is shown on Figure 3(a).
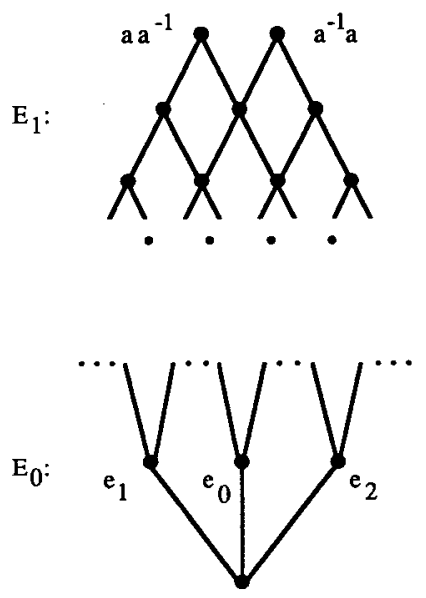

(a)
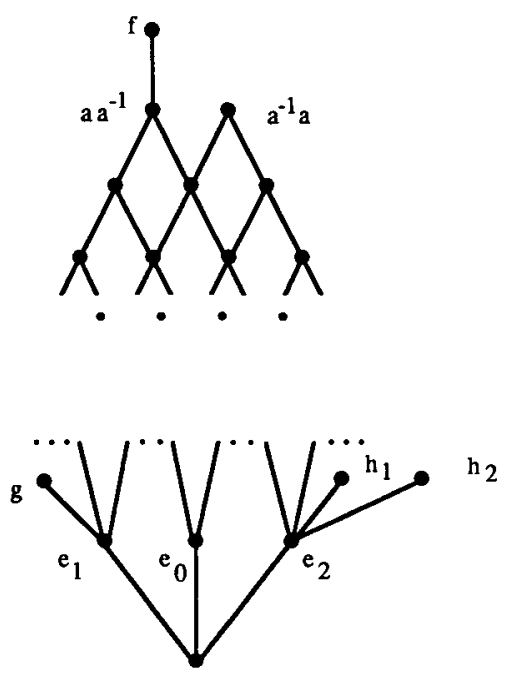

(b)

Figure 3

Let $\xi$ and $\eta$ be elements of $\mathscr{F}(\bar{E})$ such that $\left.\xi\right|_{E_{1}}=\left.\eta\right|_{E_{1}}=\theta_{a}$, and $\left.\xi\right|_{E_{0}}$ and $\left.\eta\right|_{E_{0}}$ are permutations of $E_{0}$ with the cycle decompositions $\left(e_{0} e_{1} e_{2}\right)$ and $\left(e_{0} e_{2} e_{1}\right)$, respectively. It is clear that $\xi, \eta \in T_{\bar{E}}$. Let $U$ be the full inverse subsemigroup of $T_{\bar{E}}$ generated by $\xi$. We can identify $U$ with an ideal extension of a ten-element combinatorial Brandt semigroup $B$ (having $E_{0}$ as its semilattice of idempotents) by $A^{0}$. More precisely, $U$ is identified with the semigroup whose underlying set is the (disjoint) union $A \cup B$ and whose multiplication extends that of $A$ and of $B$ and is uniquely defined by the relations: $a^{-1} e_{0} a=e_{1}, a^{-1} e_{1} a=e_{2}$ and $a^{-1} e_{2} a=e_{0}$. Let $V$ be the full inverse subsemigroup of $T_{\bar{E}}$ generated by $\eta$. Similarly to the above, we identify $V$ with the semigroup on the set $A \cup B$ whose operation * extends that of $A$ and of $B$ and is completely determined by the relations: $a^{-1} * e_{0} * a=e_{2}$, 
$a^{-1} * e_{2} * a=e_{1}$ and $a^{-1} * e_{1} * a=e_{0}$ (thus $V$ is another ideal extension of $B$ by $A^{0}$ ). Note that $E_{U}$ and $E_{V}$ have been identified with $\bar{E}$.

Now let $S$ be the ideal extension of $U$ and $T$ the ideal extension of $V$ by the five-element primitive semilattice $\left\{0, f, g, h_{1}, h_{2}\right\}$ determined by the following partial homomorphism: $f \mapsto a a^{-1}, g \mapsto e_{1}, h_{i} \mapsto e_{2}(i=1,2)$. Informally speaking, $S$ and $T$ are constructed from $U$ and $V$, respectively, by "tagging" idempotents $a a^{-1}, e_{1}$ and $e_{2}$. Denote $E_{S}\left(=E_{T}\right)$ by $E$. Then $E=\bar{E} \cup\left\{f, g, h_{1}, h_{2}\right\}$ and its diagram is given by Figure $3(\mathbf{b})$. It turns out that $S$ and $T$ are $\mathscr{P} \mathscr{A}$-isomorphic but not isomorphic combinatorial inverse semigroups. More precisely, we have

Proposition 9. Let $S$ and $T$ be the semigroups constructed in Example 1. Then

(i) $S$ and $T$ are completely semisimple combinatorial inverse semigroups;

(ii) $S$ and $T$ are not isomorphic;

(iii) $S$ and $T$ are $\mathscr{P} \mathscr{A}$-isomorphic.

Proof. (i) It is obvious that $S$ and $T$ are combinatorial. Since they do not contain inverse subsemigroups isomorphic with the bicyclic semigroup, $S$ and $T$ are completely semisimple.

(ii) Suppose that $S$ and $T$ are isomorphic and let $\phi$ be an isomorphism of $S$ onto $T$. Then $\left.\phi\right|_{E}$ is an automorphism of $E$. Since $a^{-1} a$ is a maximal element of $E$ and $a a^{-1}$ is contained in $f,\left(a a^{-1}\right) \phi=a a^{-1}$ and $\left(a^{-1} a\right) \phi=a^{-1} a$. Similarly, from the "tagging" of idempotents $e_{1}$ and $e_{2}$, we get $e_{j} \phi=e_{j}(j=$ $0,1,2)$. It follows that $\left.\phi\right|_{E}=\Delta_{E}$ and $\left.\phi\right|_{A}=\Delta_{A}$. Then

$$
e_{1}=e_{1} \phi=\left(a^{-1} e_{0} a\right) \phi=(a \phi)^{-1} * e_{0} \phi * a \phi=a^{-1} * e_{0} * a=e_{2},
$$

a contradiction. Thus $S$ and $T$ are not isomorphic.

(iii) Recall that $S$ and $T$ have the same underlying set $A \cup B \cup\left\{f, g, h_{1}, h_{2}\right\}$ and for any element of this set, its inverse in $S$ coincides with its inverse in $T$. It is straightforward to calculate the "eggbox" structure of the nonzero $\mathscr{D}$-class of $B$ in $S$ [in $T$ ] (cf. the table below).

In particular, for each $i=0,1,2, e_{i} x^{-1}=e_{i} * x$ and $x^{-1} e_{i}=x * e_{i}$ where $x \in\left\{a, a^{-1}\right\}$. Moreover, for any $n \in \mathbf{Z}$ and $i=0,1,2, a^{n} e_{i}=a^{\bar{n}} e_{i}$ and $e_{i} a^{n}=$ $e_{i} a^{\bar{n}}$ where $\bar{n} \in\{-1,0,1\}$ and $n \equiv \bar{n}(\bmod 3)$. Using this information and the standard representation for the elements of $A$ due to Gluskin (see [16, IX.1]), we can check that for all $u \in A$ and $v \in B, u * v=u^{-1} v$ and $v * u=v u^{-1}$ (note that these equalities can also be deduced from our original definition of $U$ and $V$ as full inverse subsemigroups of $T_{\bar{E}}$ generated respectively by $\xi, \eta \in T_{\bar{E}}$ such that $\left.\xi\right|_{E_{1}}=\theta_{a}=\left.\eta\right|_{E_{1}}$ and $\left.\left.\xi\right|_{E_{0}}=\left(e_{0} e_{1} e_{2}\right)=\left(\left.\eta\right|_{E_{0}}\right)^{-1}\right)$. It 


\begin{tabular}{|c|c|c|}
\hline$e_{1}$ & $e_{1} a^{-1}=a^{-1} e_{0}$ & $e_{1} a=a e_{2}$ \\
& {$\left[e_{1} * a=a * e_{0}\right]$} & {$\left[e_{1} * a^{-1}=a^{-1} * e_{2}\right]$} \\
\hline$e_{0} a=a e_{1}$ & $e_{0} a^{-1}=a^{-1} e_{2}$ \\
{$\left[e_{0} * a^{-1}=a^{-1} * e_{1}\right]$} & & \\
\hline$e_{2} a^{-1}=a^{-1} e_{1}$ & $e_{2} a=a e_{0}$ & \\
{$\left[e_{2} * a=a * e_{1}\right]$} & {$\left[e_{2} * a^{-1}=a^{-1} * e_{0}\right]$} & \\
\hline
\end{tabular}

follows, in particular, that for any $H \subseteq A \cup B \cup\left\{f, g, h_{1}, h_{2}\right\}, H \leq S$ if and only if $H \leq T$.

Take an arbitrary $\alpha \in \mathscr{P} \mathscr{A}(S)$. Note that for every $x \in S \backslash E$ if $x \in A[x \in$ $B$ ], then $\langle x\rangle$ is a free monogenic inverse [a five-element Brandt] subsemigroup of $S$. Thus if $x$ is any nonidempotent from $\operatorname{dom}(\alpha)$, then $x \alpha \in A$ if $x \in A$, and $x \alpha \in B$ if $x \in B$. Set $H=\operatorname{dom}(\alpha)$ and $K=\operatorname{ran}(\alpha)$. Take any $u, v \in H$. If $u, v \in A \cup\{f\}$ or $u, v \in B \cup\left\{g, h_{1}, h_{2}\right\}$, then

$$
(u * v) \alpha=(u v) \alpha=(u \alpha)(v \alpha)=(u \alpha) *(v \alpha) .
$$

Now suppose that $u \in A \cup\{f\}$ and $v \in B \cup\left\{g, h_{1}, h_{2}\right\}$. Then

$$
(u * v) \alpha=\left(u^{-1} v\right) \alpha=(u \alpha)^{-1}(v \alpha)=(u \alpha) *(v \alpha),
$$

and similarly $(v * u) \alpha=(v \alpha) *(u \alpha)$. Therefore $\alpha$ is an isomorphism of $(H, *)$ onto $(K, *)$, that is, $\alpha \in \mathscr{P} \mathscr{A}(T)$. Thus $\mathscr{P} \mathscr{A}(S) \subseteq \mathscr{P} \mathscr{A}(T)$ so that, by symmetry, $\mathscr{P} \mathscr{A}(S)=\mathscr{P} \mathscr{A}(T)$ and the identity mapping of $\mathscr{P} \mathscr{A}(S)$ is a $\mathscr{P} \mathscr{A}$-isomorphism of $S$ onto $T$.

REMARK 4. Another example of $\mathscr{P} \mathscr{A}$-isomorphic, but not isomorphic, combinatorial inverse semigroups can be obtained if in Example 1 we replace the free monogenic inverse semigroup $A$ by the bicyclic semigroup. This time though the corresponding nonisomorphic, but $\mathscr{P} \mathscr{A}$-isomorphic, combinatorial inverse semigroups $S$ and $T$ will not be completely semisimple.

Our next example shows that Theorem 8 need not be valid if $S$ is a shortly connected (in fact, even finite) fundamental inverse semigroup.

EXAMPLE 2. Let $E_{0}=\left\{0, e_{0}, \ldots, e_{4}\right\}$ be a six-element primitive semilattice and let $\bar{E}=E_{0}^{1}$. The diagram of $\bar{E}$ is shown in Figure 4 .

Let $\xi$ and $\eta$ be the automorphisms of $\bar{E} 1(=\bar{E})$ determined by the cycles $\left(e_{0} e_{1} e_{2} e_{3} e_{4}\right)$ and $\left(e_{0} e_{2} e_{3} e_{4} e_{1}\right)$ respectively. Let $U$ be the full inverse subsemigroup of $T_{\bar{E}}$ generated by $\xi$. Then $U$ can be identified with an ideal extension 


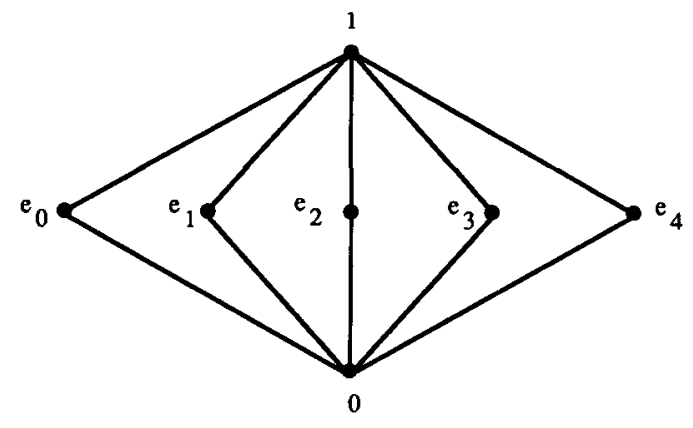

Figure 4

of a combinatorial Brandt semigroup $B$, having $E_{0}$ as its semilattice of idempotents, by $G^{0}$ where $G=\langle a\rangle$ is a cyclic group of order 5 disjoint from $B$. More precisely, $U$ is identified with the semigroup on the set $G \cup B$ whose multiplication extends that of $G$ and of $B$ and is uniquely defined by the following action of $a$ on $E_{0}: a^{-1} 0 a=0, a^{-1} e_{4} a=e_{0}$ and $a^{-1} e_{i-1} a=e_{i}$ for every $i=1, \ldots, 4$. Let $V$ be the full inverse subsemigroup of $T_{\bar{E}}$ generated by $\eta$. We identify $V$ with the semigroup on the set $G \cup B$ whose multiplication extends that of $G$ and of $B$ and is uniquely determined by the following action of $a$ on $E_{0}: a^{-1} * 0 * a=0, a^{-1} * e_{0} * a=e_{2}, a^{-1} * e_{j-1} * a=e_{j}(j=3,4)$, $a^{-1} * e_{4} * a=e_{1}$ and $a^{-1} * e_{1} * a=e_{0}$ (so that $V$ is another ideal extension of $B$ by $\left.G^{0}\right)$.

Let $S$ be the ideal extension of $U$ and $T$ the ideal extension of $V$ by the primitive semilattice $\left\{0, f_{11}, f_{12}, f_{22}, f_{13}, f_{23}, f_{33}, f_{14}, f_{24}, f_{34}, f_{44}\right\}$ determined by the following partial homomorphism: $f_{i j} \mapsto e_{j}$ for $j=1, \ldots, 4$ and $i=$ $1, \ldots, j$. Denote $E_{S}\left(=E_{T}\right)$ by $E$. We claim that $S$ and $T$ are $\mathscr{P} \mathscr{A}$-isomorphic but not isomorphic. More specifically, we have

Proposition 10. Let $S$ and $T$ be the semigroups constructed in Example 2. Then they are finite fundamental inverse semigroups which are $\mathscr{P} \mathscr{A}$ isomorphic but not isomorphic.

OUTLINE OF PROOF. It is obvious that $S$ and $T$ are finite fundamental inverse semigroups. Suppose that $S$ and $T$ are isomorphic and let $\psi$ be an isomorphism of $S$ onto $T$. From the "tagging" of idempotents, it follows that $\left.\psi\right|_{E}=\Delta_{E}$. Assume that $a \psi=a^{k}(k=1,2,3)$. Then

$$
e_{1}=\left(a^{-1} e_{0} a\right) \psi=(a \psi)^{-1} * e_{0} \psi * a \psi=a^{-k} * e_{0} * a^{k}=e_{k+1},
$$

a contradiction. Now if we suppose that $a \psi=a^{4}$, then $e_{2}=\left(a^{-1} e_{1} a\right) \psi=$ $a^{-4} * e_{1} * a^{4}=e_{4}$, again a contradiction. Therefore $S$ and $T$ are not isomorphic. 
Let $H \leq S$. It is easy to see that there are only the following possibilities: (i) $H \cap(G \backslash 1)=\varnothing$; (ii) $H=G$ or $H=G^{0}$; (iii) $G \cup B \subseteq H$. First of all, this implies that $H \leq T$, so that, $\operatorname{Sub}(S)=\operatorname{Sub}(T)$. Secondly, if $\alpha \in \mathscr{P} \mathscr{A}(S)$, $H=\operatorname{dom}(\alpha)$ and $K=\operatorname{ran}(\alpha)$, then one of the cases (i), (ii), or (iii) holds simultaneously for $H$ and for $K$, and we can show without difficulty that $\alpha \in \mathscr{P} \mathscr{A}(T)$. Thus $\mathscr{P} \mathscr{A}(S)=\mathscr{P} \mathscr{A}(T)$, so that $S$ and $T$ are trivially $\mathscr{P} \mathscr{A}$ isomorphic.

REMARK 5. The author gratefully acknowledges that he learned the general idea that led to counterexamples 1 and 2 and, in particular, the method of "tagging" idempotents, from Dr. T. E. Hall who conceived that method for one of the counterexamples in [5].

\section{Acknowledgement}

This work was done during my visits to Monash and Marquette Universities. The hospitality of the faculty and staff of the Mathematics Departments and the financial support at both institutions are appreciated very much. I am also grateful to T. E. Hall and P. R. Jones for useful discussions and comments.

\section{References}

[1] A. H. Abuhamda, 'Inductive isomorphisms of some classes of groups', Mat. Issled. 10 No. 1(35), (1975), 3-19 (in Russian).

[2] G. G. Djadchenko and B. M. Schein, 'Monogenic inverse semigroups', Algebra and Number Theory, No. 1, (1973), 3-26 (in Russian).

[3] S. M. Goberstein, 'Partial automorphisms of inverse semigroups', Proceedings of the 1984 Marquette Conference on Semigroups, (Milwaukee, Wisconsin, 1985, 29-43).

[4] S. M. Goberstein, 'Inverse semigroups determined by their bundles of correspondences', J. Algebra 125 (1989), 474-488.

[5] T. E. Hall, 'On regular semigroups II: an embedding', J. Pure Appl. Algebra 40 (1986), 215-228.

[6] J. M. Howie, An introduction to semigroup theory (Academic Press, London, 1976).

[7] K. G. Johnston, 'Lattice isomorphisms of modular inverse semigroups', Proc. Edinburgh Math. Soc. 31 (1988), 441-446.

[8] K. G. Johnston and P. R. Jones, 'Modular inverse semigroups', J. Austral. Math. Soc. (Ser. A) 43 (1987), 47-63.

[9] P. R. Jones, 'Lattice isomorphisms of inverse semigroups', Proc. Edinburgh Math. Soc. 21 (1978), 149-157.

[10] P. R. Jones, 'Distributive inverse semigroups', J. London Math. Soc. 17 (1978) 457-466.

[11] P. R. Jones, 'Lattice isomorphisms of distributive inverse semigroups', Quart. J. Math. Oxford Ser. 30 (1979), 301-314. 
[12] P. R. Jones, 'Inverse semigroups determined by their lattices of inverse subsemigroups', $J$. Austral. Math. Soc. Ser. A 30 (1981), 321-346.

[13] A. L. Libih, 'On the theory of inverse semigroups of local automorphisms', Theory of semigroups and its applications, no. 3, pp. 46-59 (Saratov Univ. Press, 1974, in Russian).

[14] A. L. Libih, 'Local automorphisms of commutative monomorphic inverse semigroups', Studies in algebra, no. 4, pp. 56-69 (Saratov Univ. Press, 1974, in Russian).

[15] A. L. Libih, Inverse semigroups of local automorphisms of commutative semigroups, (Ph.D. Dissertation Summary, Saratov State University, 1974, 12 pp., in Russian).

[16] M. Petrich, Inverse semigroups (Wiley, New York, 1984).

[17] G. B. Preston, 'Inverse semigroups: some open questions', Proc. Symposium on Inverse Semigroups and their Generalizations, pp. 122-139 (Northern Illinois Univ., 1973).

[18] E. L. Sadovskii, 'Lattice isomorphisms of free groups and free products', Mat. $S b$. 14 (56) (1944), 155-173 (in Russian).

[19] B. M. Schein, 'An idempotent semigroup is determined by the pseudogroup of its local automorphisms', Mat. Zap. Ural. Univ. 7 No. 3, (1970), 222-233 (in Russian).

[20] L. N. Shevrin and A. J. Ovsyannikov, 'Semigroups and their subsemigroup lattices', Semigroup Forum 27 (1983), 1-154.

\section{Department of Mathematics \\ California State University \\ Chico, California 95929 \\ U.S.A.}

